\title{
Discussion on Strain Accommodation Associated with Formation of LPSO Structure
}

\author{
Tadashi Furuhara* and Xinfu Gu \\ Institute for Materials Research, Tohoku University, Sendai 980-8577, Japan
}

\begin{abstract}
Plate-shaped precipitates accompanying change of chemical composition/order exhibit characteristics of displacive transformation when stacking sequence changes. One of intrinsic nature of long-period stacking ordered (LPSO) structures is, regular arrangements of stacking faults should cause significant change in local elastic strain field. This study intends to discuss problems of elastic strain in associated with LPSO structure and plastic accommodation processes by diffusion. It is indicated that required time for strain accommodation through boundary diffusion is negligibly small whereas that for volume diffusion is comparable to aging time where LPSO structure are formed via precipitation from the supersaturated Mg matrix. However, high degree of coherency between the fcc-base structural unit in the LPSO structure and Mg matrix, high-speed diffusion path for diffusional accommodation cannot be provided and thus, the volume diffusion controlled process or selfaccommodation by combination of structural units with alternative shears should be dominant. The observation of $14 \mathrm{H}$ or $10 \mathrm{H}$-type LPSO structure by aging clearly indicates such strain accommodation during sequence of precipitation. [doi:10.2320/matertrans.MI201224]
\end{abstract}

(Received December 11, 2012; Accepted January 24, 2013; Published March 15, 2013)

Keywords: precipitation, interface, diffusion, strain energy, long period stacking ordered structure

\section{Introduction}

It has been well known that plate-shaped precipitates accompanying change of chemical composition/order exhibit characteristics of displacive transformation when stacking sequence changes. ${ }^{1,2)}$ Such a transformation takes place by the motion of interfacial defects with partial dislocation and/ or step character. Those defects were denoted as structural/ growth ledges or transformation dislocations. ${ }^{1,3)}$ Later, more general concept, i.e., transformation disconnection, was proposed. ${ }^{4,5)}$

Figure 1 shows schematically the atomic motion in front of a transformation disconnection. ${ }^{6}$ In a diffusionless/ displacive transformation (i.e., martensitic transformation), the motion of a single atom can be described by small displacement from the matrix lattice site (M) to the product lattice site $(\mathrm{P})$, as is schematically shown in Fig. 1(a). Such a displacement is described as the Burgers vector associated with the transformation disconnection. However, when the partitioning of substitutional alloying elements occurs, diffusion aided by motion of vacancies around the interface needs to be involved. When a vacancy (V) is placed at a matrix site right next to the interface (Fig. 1(b)), several matrix atoms (A, B and $\mathrm{C}$ in the figure) are able to jump into this site. However, the position of the adjacent site in the product phase $(\mathrm{P})$ is so close to the vacancy site in the matrix. Thus, a matrix atom can jump directly into the product lattice site (Fig. 1(c)). In such a case, there are several paths for such diffusional jumps though each of them may not have the same activation energy. Although there is no one-to-one atomic correspondence, the lattice (or atomic) site correspondence ${ }^{7)}$ accurately maintained since these jumps can be divided virtually into two components (a diffusional jump in the matrix lattice and a small displacement or shuffle from the matrix site to the product site).

The presence of one-to-one lattice site correspondence between matrix and precipitate phases results in requirement

*Corresponding author, E-mail: furuhara@imr.tohoku.ac.jp (a)

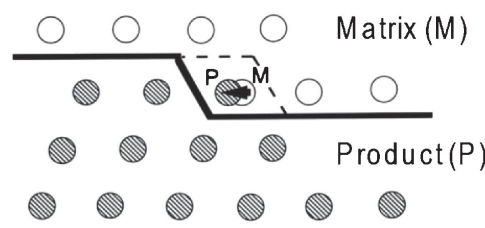

(b)

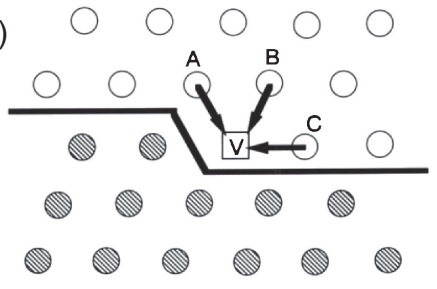

(c)

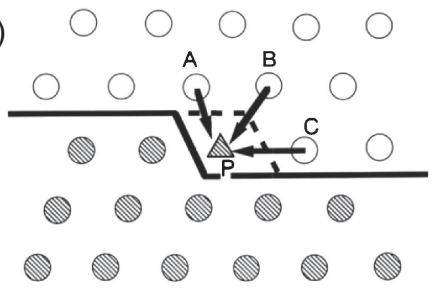

Fig. 1 Atomic site correspondence with migration of transformation disconnection at interphase boundary between matrix $(\mathrm{M})$ and product (P) phases; (a) displacement of atoms by shear, (b) diffusional jumps in matrix at growth front, (c) diffusional jump from matrix lattice sites directly into a product lattice site.

of strain accommodation. There are several kinds of accommodation mechanisms. ${ }^{2}$ ) Figure 2(a) shows lattice deformation provided by pure shear. Suppose that matrix region enclosed by the dashed line in (a) is transformed by such shear without constraint from the surrounding region. Total numbers of atoms are conserved in this transformation. After the deformation, the white area becomes less in atomic density whereas the other side becomes more densely packed. Elastic accommodation first takes place but soon plastic accommodation comes in play during the growth of the 
(a)

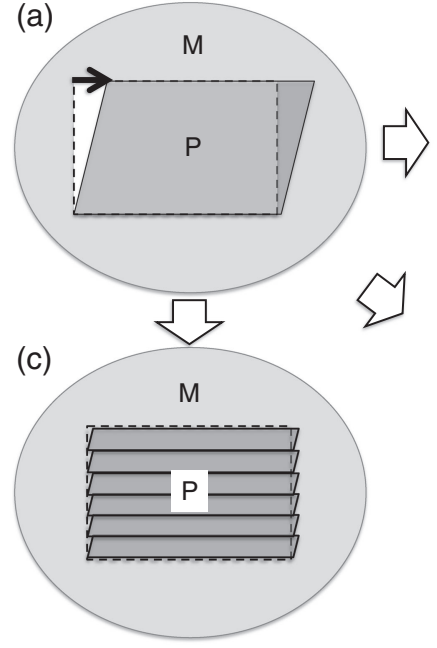

(b)

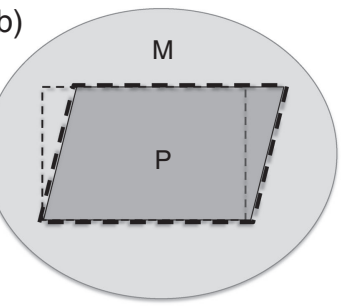

(d)

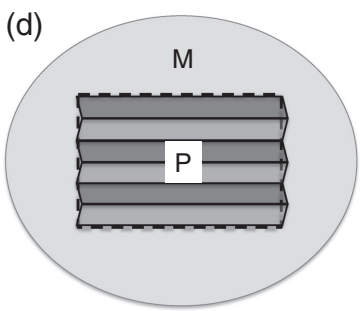

Fig. 2 Schematic diagrams of accommodation of transformation strain (pure shear component); (a) shear strain in transformation, (b) plastic accommodation in matrix, (c) plastic accommodation in product phase by dislocation slip and (d) by twinning or formation of stacking faults accompanying shear.

product phase. Plastic deformation can occur both in matrix and product phase via diffusion or deformation by slip or twinning. In Fig. 2(b), the accommodation in the matrix is described. The atomic density is recovered both in the left and right region of the product. This can be achieved by diffusion of matrix atoms from the right to the left either by bulk or interfacial diffusion. Figures 2(c) and 2(d) show that macroscopic shape of the product phase is maintained to be the same as that of the original matrix. In (c), plastic deformation of product phase occurs by dislocation slip whereas opposite shear is introduced, for example, by twinning in (d), as typically observed in ferrous martensite. Such accommodation is often seen in precipitation processes accompanying shear displacement. ${ }^{8)}$ When volume change is accompanied, long-range diffusion is needed for complete relaxation.

On the formation mechanisms of long-period stacking ordered (LPSO) structure, regular arrangement of stacking faults with solute enrichment has been recognized. ${ }^{9)}$ Since they provide local accumulation of strain (mostly of shear component), it is necessary to consider strain accommodation processes associated with the LPSO structures. Recently, a bi-atomic layer high stacking fault was re-examined as a fourlayer high fec structural unit with chemical ordering and two categories of LPSO structures were identified in arrangement of the structural units. ${ }^{10,11)}$ However, mechanisms and kinetics of strain accommodation should be examined further in detail. This study intends to discuss feasibility of plastic accommodation processes by diffusion because alloy partitioning between the LPSO structure and Mg matrix requires long-range diffusion.

\section{Transformation Disconnections in fec $\leftrightarrow$ hep Trans- formation}

It has been well known that transformation disconnections in $\mathrm{fcc} \leftrightarrow \mathrm{hcp}$ transformation are Shockley partial dislocations. ${ }^{12)}$ For example, in precipitation of hcp $\gamma^{\prime}$ phase in the

(a)

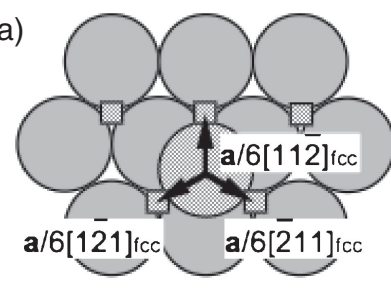

(111)fcc (b)

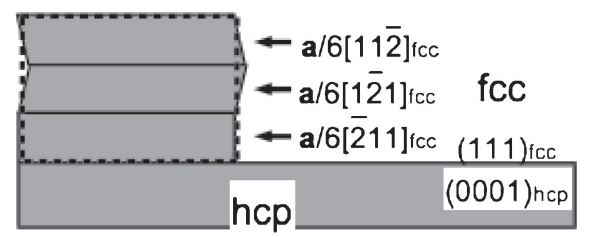

Fig. 3 Description of transformation disconnection in $\mathrm{fcc} \rightarrow \mathrm{hcp}$ transformation; (a) three equivalent shears associated with Shockley partial on $(111)_{\text {fcc }}$ plane, (b) alternative operation of the three partials resulting in mutual cancellation of transformation shear strain.

fcc $\alpha$ matrix of an Al-Ag alloy, the disconnection playing a role of growth ledge exists on the broad face of precipitate plates with the habit plane of $(111)_{\text {fcc }} / /(0001)_{\text {hcp. }}$. Howe et al. ${ }^{13)}$ showed that a unit growth ledge contains a a/ $6\langle 112\rangle_{\mathrm{fcc}}$ Shockley partial dislocation, promotes the stacking sequence change from fcc to hcp structure. Figure 3(a) shows schematically atomic displacement associated with Shockley partials on (111) fec plane. ${ }^{12)}$ By shifting atoms on the B and C layers to the $\mathrm{C}$ and $\mathrm{A}$ layers, respectively, two layers of hcp structure are created. Operation of a Shockley partial every other (111) fcc layer leads to thickening of hcp phase in the fcc matrix. However, to form a single variant of hcp phase from the fcc matrix, any of three possible partials $\left(\mathbf{a} / 6[11 \overline{2}]_{\mathrm{fcc}}\right.$, $\mathbf{a} / 6[1 \overline{2} 1]_{\mathrm{fcc}}$ and $\mathbf{a} / 6[\overline{2} 11]_{\mathrm{fcc}}$ ) on the same (111) fcc plane can glide on every other atomic plane as seen in Fig. 3(a). Figure 3(b) shows that, if the same amount of each partial dislocation operates, the macroscopic shear strain is zero. The HRTEM micrograph of Fig. 4 shows the atomic structure of the edge of a $\gamma^{\prime}$ plate in an Al-15 mass\% Ag alloy. ${ }^{14)}$ This interface is formed by the coalescence of many growth ledges. By drawing Burgers circuits across this interface at various positions, it is easy to know that the three partials operate alternately in the growth of this precipitate plate. Thus, self-accommodation of transformation shear strain occurs in this transformation. On the other hand, volumetric strain is again accommodated by sessile misfit dislocations of $\mathbf{b}=\mathbf{a} / 3[111]_{\mathrm{fcc}}=\mathbf{a} / 2[0001]_{\mathrm{hcp}}$ (the arrow in Fig. 4). This result coincides well with that of Howe et al. ${ }^{13)}$

A bi-atomic layer high stacking fault in LPSO structure needs to contain a partial dislocations in terms of hcp Mg, equivalent to a Shockley partial in fcc structure, at its growth front. Recently, Nie and his co-workers ${ }^{10,11)}$ studied precipitation processes in $\mathrm{Mg}-(\mathrm{Gd}, \mathrm{Y})-\mathrm{Zn}$ alloys and demonstrated that originally proposed bi-atomic layer high stacking fault can be regarded as four-layer high fcc structure with chemical ordering. Although the sequence of changes in stacking and chemical composition/order is still under discussion, it is clear that the stacking change with the formation of LPSO structure accompanies large shear strain essentially. 

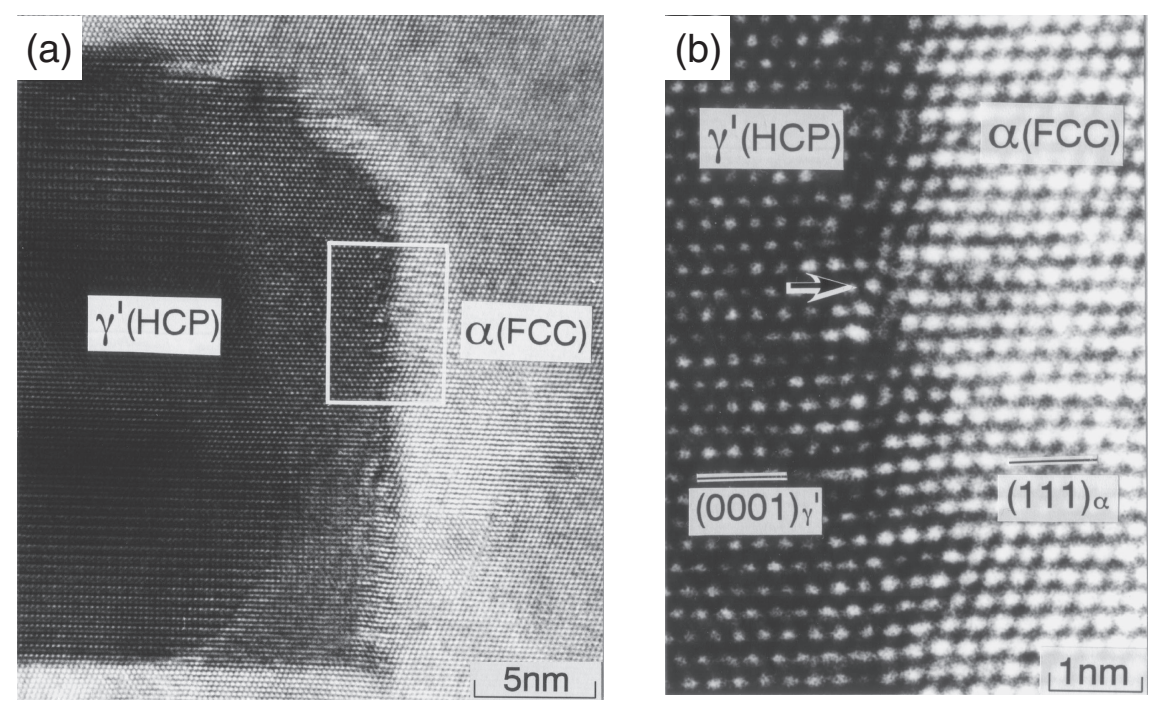

Fig. 4 High-resolution transmission electron micrographs showing interphase boundary structures between $\gamma^{\prime \prime}$ and fcc matrix in an Al15 mass\% Ag alloy: ${ }^{14)}$ (a) a macroscopic view of the edge of $\gamma^{\prime \prime}$ plate, (b) an enlargement of the edge revealing that there is no accumulation of shear strain.

Table 1 Property parameters of pure magnesium used in calculation. ${ }^{16,17)}$

\begin{tabular}{|c|c|c|c|c|c|c|}
\hline $\begin{array}{l}\text { Atomic } \\
\text { volume } \\
\Omega / \mathrm{m}^{3}\end{array}$ & $\begin{array}{c}\text { Shear modulus } \\
\mu / \mathrm{MN} \cdot \mathrm{m}^{-2} \\
(300 \mathrm{~K})\end{array}$ & $\begin{array}{l}\text { Poisson's } \\
\text { ratio } v\end{array}$ & $\begin{array}{l}\text { Volume } \\
\text { diffusion } \\
D_{0} / \mathrm{m}^{2} \mathrm{~s}^{-1}\end{array}$ & $\begin{array}{c}\text { Volume } \\
\text { diffusion } \\
Q / \mathrm{kJ}^{-1} \mathrm{~mol}^{-1}\end{array}$ & $\begin{array}{c}\text { Grain boundary } \\
\text { diffusion } \\
D_{0} \delta / \mathrm{m}^{3} \cdot \mathrm{s}\end{array}$ & $\begin{array}{c}\text { Grain boundary } \\
\text { diffusion } \\
Q / \mathrm{kJ} \cdot \mathrm{mol}^{-1}\end{array}$ \\
\hline $2.33 \mathrm{E}-28$ & $1.66 \mathrm{E}+04$ & 0.29 & $1.0 \mathrm{E}-04$ & 135 & $5.0 \mathrm{E}-12$ & 92 \\
\hline
\end{tabular}

Table 2 Calculated relaxation time by diffusional process for different temperature and size of precipitate.

\begin{tabular}{|c|c|c|c|c|c|c|}
\hline \multirow{2}{*}{$\begin{array}{l}\text { Particle } \\
\text { radius, } \\
\quad a\end{array}$} & \multicolumn{3}{|c|}{$\begin{array}{l}\text { Relaxation time via boundary diffusion, } \\
\qquad \tau_{\mathrm{b}} / \mathrm{s}\end{array}$} & \multicolumn{3}{|c|}{$\begin{array}{l}\text { Relaxation time via volume diffusion, } \\
\qquad \tau_{\mathrm{v}} / \mathrm{s}\end{array}$} \\
\hline & $423 \mathrm{~K}$ & $573 \mathrm{~K}$ & $773 \mathrm{~K}$ & $423 \mathrm{~K}$ & $573 \mathrm{~K}$ & $773 \mathrm{~K}$ \\
\hline $1 \mathrm{~nm}$ & $2.12 \mathrm{E}-14$ & $3.12 \mathrm{E}-14$ & $4.78 \mathrm{E}-14$ & $2.27 \mathrm{E}+05$ & $1.46 \mathrm{E}+01$ & $1.47 \mathrm{E}-02$ \\
\hline $10 \mathrm{~nm}$ & $2.12 \mathrm{E}-11$ & $3.12 \mathrm{E}-11$ & $4.78 \mathrm{E}-11$ & $2.27 \mathrm{E}+07$ & $1.46 \mathrm{E}+03$ & $1.47 \mathrm{E}+00$ \\
\hline $100 \mathrm{~nm}$ & $2.12 \mathrm{E}-08$ & $3.12 \mathrm{E}-08$ & $4.78 \mathrm{E}-08$ & $2.27 \mathrm{E}+09$ & $1.46 \mathrm{E}+05$ & $1.47 \mathrm{E}+02$ \\
\hline
\end{tabular}

\section{Possibility of Diffusional Relaxation}

As was mentioned in the introduction, diffusion associated with precipitation can significantly contribute to relaxation of transformation strain. Onaka and Kato ${ }^{15)}$ discussed kinetics of relaxation process by diffusion. According to them, relaxation times for boundary diffusion and volume diffusion, $\tau_{\mathrm{b}}$ and $\tau_{\mathrm{v}}$ are expressed as follows, respectively, by assuming that particle shape is spherical and two phases have the same isotropic elastic property:

$$
\begin{aligned}
\tau_{\mathrm{b}} & =\frac{1-v}{2(7-5 v) \mu} \frac{k T a^{3}}{D_{\mathrm{b}} \delta \Omega} \\
\tau_{\mathrm{V}} & =\frac{3-5 v}{4(1+v) \mu} \frac{k T a^{2}}{D_{\mathrm{V}} \Omega}
\end{aligned}
$$

where $a$ : particle radius, $v$ : Poisson's ratio of the matrix, $\mu$ : shear modulus of the matrix, $k$ : the Boltzmann constant, $T$ : temperature, $D_{\mathrm{b}}$ and $D_{\mathrm{v}}$ : boundary and volume diffusion coefficients, $\delta$ : boundary thickness and $\Omega$ : atomic volume, respectively. Boundary diffusion leads to accommodation of shear strain and resultant hydrostatic stress field whereas nonconservative atomic motion promoted by volume diffusion between the interfacial region and sinks/sources of vacancies (e.g., matrix grain boundaries) is necessary to have complete relaxation of both shear and volumetric stress components. By substituting appropriate data for pure $\mathrm{Mg}$ listed in Table $1,{ }^{16,17)}$ the relaxation times for both cases were calculated. Table 2 shows the estimated time in a range of particle size suitable for LPSO structure. It was confirmed that required time for strain accommodation through boundary diffusion is negligibly small. Based upon this calculation, it appears that plastic accommodation of shear strain by diffusion can be easily established.

However, according to the Precipitation-Time-Temperature (PTT) diagram reported by Yamasaki et al., ${ }^{18)} \gamma^{\prime \prime} \rightarrow 14 \mathrm{H}$ transition in a Mg-Zn-Gd system occurs after holding of $1 \mathrm{~h}$ at $573 \mathrm{~K}$ and several minutes at $773 \mathrm{~K}$. The size of LPSO structure is in orders of $10-100 \mathrm{~nm}$ in thickness. The relaxation time for volume diffusion is comparable to the observed aging time required for formation of $14 \mathrm{H} \mathrm{LPSO}$ structure. This could be understood in terms of coherency between the fcc $\gamma^{\prime \prime}$ phase and the hcp Mg matrix. The $(0001)_{\mathrm{hcp}} / /(111)_{\mathrm{fcc}}$ plane, which is the habit plane of $\gamma^{\prime \prime}$ plate is a lattice invariant plane in nature because of equivalent 
atomic configurations. High-speed path for diffusional accommodation cannot be provided along such a boundary of good coherency and thus, the volume diffusion controlled process or self-accommodation should be dominant. For the latter case, structural units of opposite shears tend to be formed neighboring to each other as an ordinary precipitation sequence. $14 \mathrm{H}$ and $10 \mathrm{H}$ structures contain such alternative arrangements of structural units. This suggests that different Shockley partials operate in those LPSO structures formed by aging. In contrast, both of $18 \mathrm{R}$ and $24 \mathrm{R}$ structures contain fcc structural units of the same shear. Since $18 \mathrm{R}$ structure is rarely observed in aged specimens, local stress arising in solidification enable preferred formation of structural units of the single shear.

To understand consecutive formation of fcc structural units in LPSO structures, elastic interaction between neighboring structural units needs to be examined.

\section{Strain Associated with Formation of LPSO Structure}

In this section, we would like to discuss the strain associated with LPSO structure based upon Eshelby's method. ${ }^{19)}$

The shear strain associated with the four-layer high fcc structure is about 0.089 . The elastic strain energy will be calculated for different aspect ratio. Because Magnesium is nearly elastically isotropic, ${ }^{20)}$ the system is assumed to be elastic homogeneous and isotropic for simplicity, and the elastic modulus is shown in Table 1. The inclusion or precipitate is assumed to be of cuboidal shape. The cuboidal size is defined as $2 b_{1}, 2 b_{2}$ and $2 b_{3}$ along $\boldsymbol{x} / /[100], \boldsymbol{y} / /[010]$, $\boldsymbol{z} / /[001]$ respectively, and $b_{1}=b_{2}$. The aspect ratio is defined as $\beta=b_{3} / b_{1}$. The elastic strain energy for different aspect ratio and modulus $\left(\mu^{*}=0.3 \mu, \mu^{*}=\mu, \mu^{*}=3 \mu\right)$ has been calculated by Eshelby's method ${ }^{19)}$ as shown in Fig. 5, where $\mu^{*}$ is the elastic modulus for precipitate. The different shear modulus applied here is going to take the chemical ordering effect in LPSO structure into account. When the aspect ratio is 0.01 , the elastic strain energy is smaller than $1000 \mathrm{~J} / \mathrm{mol}$. As the aspect ratio becomes larger in Fig. 5, the elastic energy for different shear modulus increases rapidly when the aspect ratio is smaller than 0.5 . Thus, thickening of precipitate is energetically unfavorable despite of the chemical ordering in LPSO. Therefore, the growth of precipitate with the same shear direction is nearly impermissible. The interaction of the plate-shaped precipitate with different shear direction is considered as following.

Suppose the infinite space contains two elastic homogeneous and isotropic precipitates $\Omega_{1}$ and $\Omega_{2}$ with the transformation strain (eigenstrain) as $\varepsilon^{(1)}$ and $\varepsilon^{(2)}$ receptively. The elastic strain energy of the system is ${ }^{19)}$

$E=-\frac{1}{2}\left[\int_{\Omega 1} \sigma^{(1)} \varepsilon^{(1)} \mathrm{d} \Omega+\int_{\Omega 2} \sigma^{(2)} \varepsilon^{(2)} \mathrm{d} \Omega+2 \int_{\Omega 1} \sigma^{(2)} \varepsilon^{(1)} \mathrm{d} \Omega\right]$

where $\sigma^{(1)}$ is the stress caused by $\varepsilon^{(1)}$ and $\sigma^{(2)}$ is the stress caused by $\varepsilon^{(2)}$. Since the elastic energy of a single precipitate in an infinite space is equal to $E_{\text {single }}=-\frac{1}{2} \int_{\Omega} \sigma \varepsilon \mathrm{d} \Omega,{ }^{19)}$ the interaction energy between $\Omega_{1}$ and $\Omega_{2}$ is

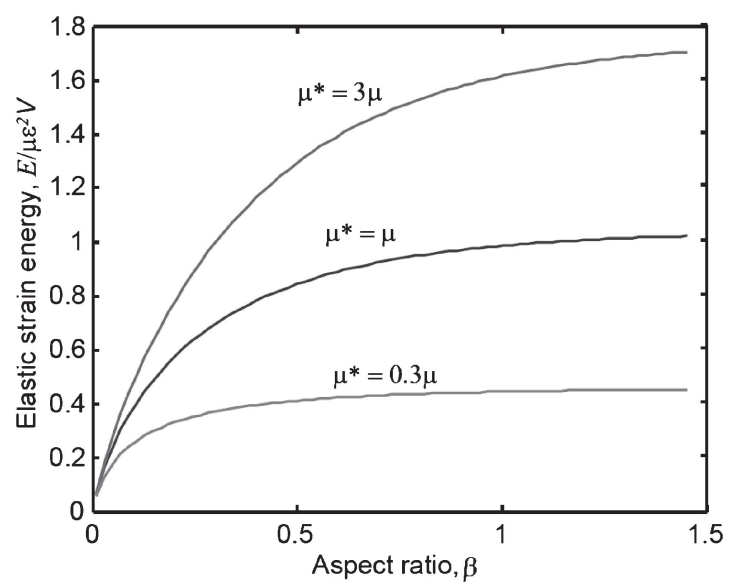

Fig. 5 Variation of the elastic strain energy with different aspect ratio and elastic modulus for a cuboidal shaped precipitate. The energy is scaled by $\mu \varepsilon^{2} V$ where $V$ is the volume of the precipitate and $\varepsilon$ is the magnitude of shear strain (Same for the other figures).

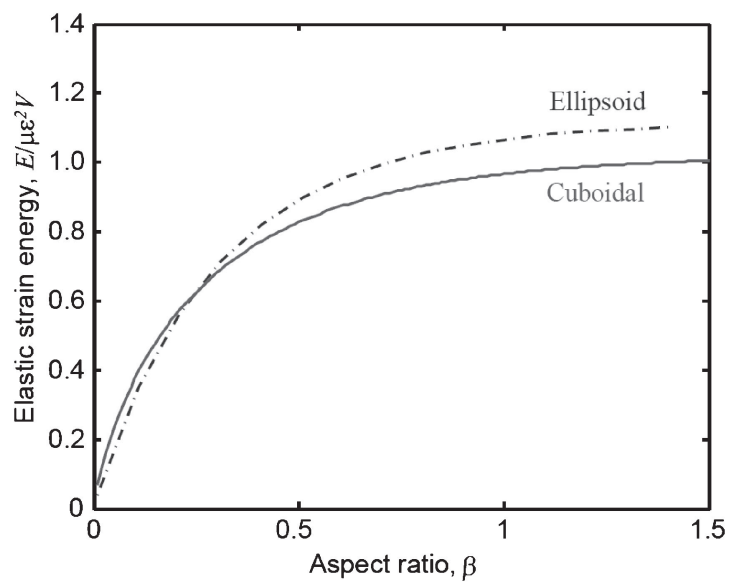

Fig. 6 Variation of the elastic strain energy with different aspect ratio for an ellipsoidal or cuboidal shaped precipitate.

$$
E_{\text {int }}=E+\frac{1}{2} \int_{\Omega 1} \sigma^{(1)} \varepsilon^{(1)} \mathrm{d} \Omega+\frac{1}{2} \int_{\Omega 2} \sigma^{(2)} \varepsilon^{(2)} \mathrm{d} \Omega .
$$

By substituting eq. (3) into eq. (4), eq. (4) becomes

$$
E_{\text {int }}=-\int_{\Omega 1} \sigma^{(2)} \varepsilon^{(1)} \mathrm{d} \Omega \text {. }
$$

For a given system, the strain is usually known, but the stress field is often complex. For the cuboidal case, the stress fields are explicitly expressed in Ref. 21), and eq. (5) can be easily calculated. Therefore, the interaction between two cuboids of the same size is considered in this study.

The elastic strain energy for a single ellipsoid or cuboidal shaped precipitate in the infinite space is shown in Fig. 6. The elastic energy in both cases increases monotonically with the aspect ratio. The elastic energy difference between two shapes is small, especially when the aspect ratio is small. The energy value in Fig. 6 will be used to compare with the interaction energy.

The overall minimum elastic strain interaction energy is reached when the shear strain of two precipitates has the opposite sign, and the precipitates closely stack with broad face to face. For example, Fig. 7 shows the variation of the interaction energy with the distance along [001] direction for 


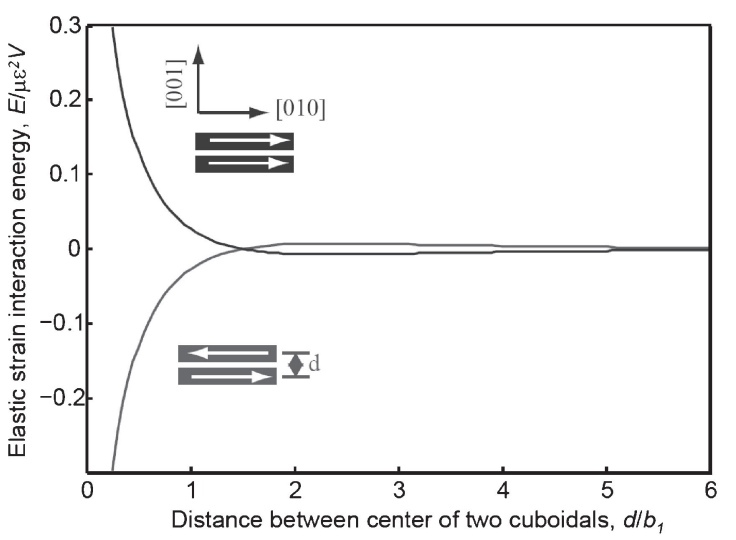

Fig. 7 Variation of the interaction energy with the distance $d$ between two precipitates along [001] direction when $\beta=0.1$. The red curve is for the precipitate with opposite shear direction (see inserted figure), while the blue curve is for the same shear direction.

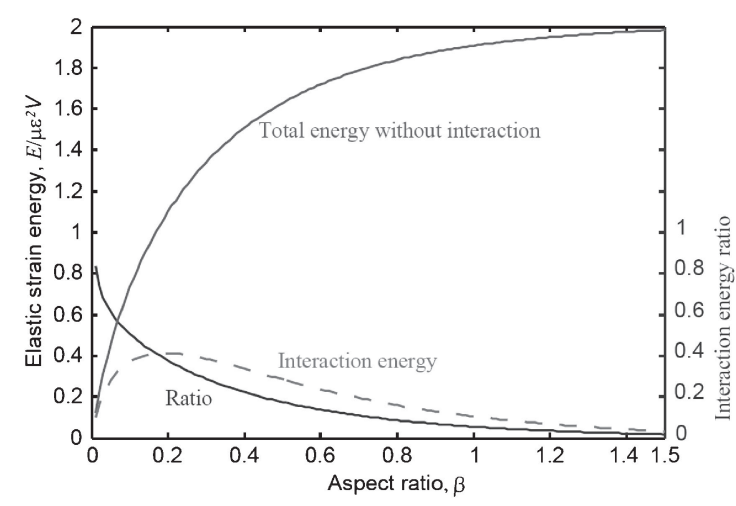

Fig. 8 Variation of the interaction energy, the elastic energy without interaction and their ratio with the aspect ratio.

two cases when $\beta=0.1$. One case shows the two precipitates with same shear direction and the other is for the two precipitates with opposite shear direction. The interaction energy cures for two cases are symmetrical as expected from eq. (5). Only the latter case has negative interaction energy, and it is energetically favorable. The interaction energy curve has a minimum, when the center distance is $2 b_{3}$ i.e., two precipitates contact. Figure 8 shows the variation of the elastic energy, the interaction energy and their ratio with the aspect ratio. The elastic strain energy in Fig. 8 is the energy when the interaction energy is not considered, i.e., the first two terms of eq. (3). The energy ratio in this figure shows the percentage of the energy released due to the interaction. When the aspect ratio increases, the interaction becomes weak. Thus, when the aspect ratio is small, the interaction energy should not be neglected. As shown in Fig. 8, the interaction energy could be as high as about $80 \%$ of overall elastic energy $\left(2 E_{\text {single }}\right)$, i.e., $1.6 E_{\text {single. }}$ That is to say the elastic strain energy for formation of an opposite precipitate is fully accommodated, and the extra energy (about $0.6 E_{\text {single }}$ ) is available to be the driving force.

Therefore, from the point view of elastic energy interaction, it is confirmed that the energetically favorable LPSO structure is the one containing structural units with opposite shear neighboring to each other, such as $10 \mathrm{H}$ or $14 \mathrm{H}$ type. However, to examine origins of the variation in number of hcp Mg layers inserted between neighboring fcc structural units, it is presumably necessary to examine chemical interaction between those units in future.

\section{Conclusion}

In this paper, transformation strain and feasibility of plastic accommodation by diffusion-controlled processes were discussed for formation of the LPSO structure in $\mathrm{Mg}$ matrix. The main conclusions are summarized in the following.

It is indicated that required time for strain accommodation through boundary diffusion is negligibly small whereas that for volume diffusion is comparable to aging time where LPSO structure are formed via precipitation from the supersaturated $\mathrm{Mg}$ matrix. However, because of coherency between the fcc-base structural unit in the LPSO structure and $\mathrm{Mg}$ matrix, slow accommodation kinetics mainly controlled by the volume diffusion or self-accommodation by combination of structural units with alternative shears should be dominant. The observation of $14 \mathrm{H}$ or $10 \mathrm{H}$-type LPSO structure by aging clearly indicates such strain accommodation during sequence of precipitation.

\section{Acknowledgments}

This work was supported by MEXT KAKENHI Grant Number 23109006 (Grant-in-Aid for Scientific Research on Innovative Areas, "Synchronized Long-Period Stacking Ordered Structure - The Evolution of the Material Science for Innovative Development of the Next-generation Lightweight Structure Materials -").

\section{REFERENCES}

1) H. I. Aaronson, T. Furuhara, J. M. Rigsbee, W. T. Reynolds, Jr. and J. M. Howe: Metall. Trans. A 21 (1990) 2369-2409.

2) J. W. Christian: Metall. Mater. Trans. A 25 (1994) 1821-1839.

3) B. A. Bilby: Phil. Mag. 44 (1953) 782-785.

4) J. P. Hirth and R. C. Ponds: Acta Mater. 44 (1996) 4749-4763.

5) H. I. Aaronson, B. C. Muddle, J. F. Nie and J. P. Hirth: Metall. Mater. Trans. A 33 (2002) 2541-2547.

6) T. Furuhara, K. Wada and T. Maki: Metall. Mater. Trans. A 26 (1995) 1971-1978.

7) J. M. Howe: Metall. Mater. Trans. A 25 (1994) 1917-1922.

8 ) B. C. Muddle, J. F. Nie and G. R. Hugo: Metall. Mater. Trans. A 25 (1994) 1841-1856.

9) E. Abe, Y. Kawamura, K. Hayashi and A. Inoue: Acta Mater. 50 (2002) 3845-3857.

10) J. F. Nie, K. Oh-ishi, X. Gao and K. Hono: Acta Mater. 56 (2008) 6061-6076.

11) Y. M. Zhu, M. Weyland, A. J. Morton, K. Oh-ishi, K. Hono and J. F. Nie: Scr. Mater. 60 (2009) 980-983.

12) T. Furuhara and T. Maki: Scr. Mater. 34 (1996) 929-934

13) J. M. Howe, U. Dahmen and R. Gronski: Phil. Mag. A 56 (1987) 31-61.

14) T. Furuhara and T. Maki: Materia Japan 36 (1997) 483-490.

15) S. Onaka and M. Kato: Mater. Sci. Eng. A 146 (1991) 217-232.

16) Metals Data Book, ed. by Japan Inst. Metals, (Maruzen Co., Ltd., 1984) p. 1.

17) H. J. Frost and M. J. Ashby: Deformation-Mechanism Maps, (Pergamon Press, Oxford, 1982) p. 43.

18) M. Yamasaki, M. Sasaki, M. Nishijima, K. Hiraga and Y. Kawamura: Acta Mater. 55 (2007) 6798-6805.

19) T. Mura: Micromechanics of Defects in Solids, (Martinus Nijhoff Pub., Dordrecht, 1987).

20) L. J. Slutsky and C. W. Garland: Phys. Rev. 107 (1957) 972-976.

21) Q. Li and P. Anderson: J. Elast. 64 (2001) 237-245. 